\title{
ON SOME DETERMINANTAL INEQUALITIES
}

\section{DAVID CARLSON ${ }^{1}$}

1. We shall discuss two recent theorems by Marvin Marcus ([6, Theorem 3]) and Ky Fan ([4, Theorem 1]) involving related determinantal inequalities. We give improvements of their inequalities, and show how they follow from the previously known special cases of the theorems.

We deal with real or complex matrices $A$ of order $n$. For any subset $\beta$ of $\{1, \cdots, n\}$, we denote by $A(\beta)$ the principal minor on the rows and columns of $A$ indexed by $\beta$; clearly the order in which the indices of $\beta$ occur is irrelevant. We define $A(\varnothing)=1$. Now let $\beta_{1}, \cdots, \beta_{k}$ be subsets of $\{1, \cdots, n\}$, and $h$ a positive integer between 1 and $k$; we define

$$
\phi\left(A ; h ; \beta_{1}, \cdots, \beta_{k}\right)=(\operatorname{det} A)^{h} / A\left(\beta_{1}\right) \cdots A\left(\beta_{k}\right),
$$

if $A\left(\beta_{i}\right) \neq 0, i=1, \cdots, k$. We may now state the theorems mentioned above.

Theorem A (Marcus). Let $A$ be positive definite hermitian, and $\beta_{1}, \cdots, \beta_{k}$ subsets of $\{1, \cdots, n\}$.

Suppose each $i, i=1, \cdots, n$, occurs in exactly $h$ of the $\beta_{j}$. Then

$$
\phi\left(A ; h ; \beta_{1}, \cdots, \beta_{k}\right) \leqq 1 .
$$

A proof of Theorem A for totally positive (i.e., all minors positive) and (nonsingular) $M$-matrices (cf. [7] or [3]) was given by Fan in $[4]$.

Theorem B (FAN). Let $A$ be a nonsingular $M$-matrix and $B$ any complex matrix for which

$a_{i i} \leqq\left|b_{i i}\right|, i=1, \cdots, n ;\left|b_{i j}\right| \leqq\left|a_{i j}\right|, i, j=1, \cdots, n ; i \neq j$. Let $\beta_{1}, \cdots, \beta_{k}$ be subsets of $\{1, \cdots, n\}$. If each $i, i=1, \cdots, n$, occurs in at most $h$ of the $\beta_{j}$, then

$$
\phi\left(A ; h ; \beta_{1}, \cdots, \beta_{k}\right) \leqq\left|\phi\left(B ; h ; \beta_{1}, \cdots, \beta_{k}\right)\right| .
$$

2. Now for our theorems. Given $\beta_{1}, \cdots, \beta_{k}$, we define, for $h$ $=1, \cdots, k$,

Presented to the Society, November 19, 1966; received by the editors January 18, 1967.

${ }^{1}$ Research supported by the National Science Foundation, Grant GP-4051. 


$$
\alpha_{h}=\left\{i \mid i \text { occurs in at least } h \text { of the } \beta_{j}\right\} .
$$

We then define

$$
\psi\left(A ; \beta_{1}, \cdots, \beta_{k}\right)=A\left(\alpha_{1}\right) \cdots A\left(\alpha_{k}\right) / A\left(\beta_{1}\right) \cdots A\left(\beta_{k}\right),
$$

(again, if $\left.A\left(\beta_{i}\right) \neq 0, i=1, \cdots, k\right)$. If $k=2$, we have

$$
\psi\left(A ; \beta_{1}, \beta_{2}\right)=\frac{A\left(\alpha_{1}\right) A\left(\alpha_{2}\right)}{A\left(\beta_{1}\right) A\left(\beta_{2}\right)}=\frac{A\left(\beta_{1} \cup \beta_{2}\right) A\left(\beta_{1} \cap \beta_{2}\right)}{A\left(\beta_{1}\right) A\left(\beta_{2}\right)} .
$$

TheOREM 1. Suppose all principal minors of $A$ are nonzero and $\left|\psi\left(A ; \beta_{1}, \beta_{2}\right)\right| \leqq 1$ for all $\beta_{1}, \beta_{2}$. Then, for all $\beta_{1}, \cdots, \beta_{k}$,

$$
\left|\psi\left(A ; \beta_{1}, \cdots, \beta_{k}\right)\right| \leqq 1 .
$$

If each $i$ occurs in exactly $h$ of the $\beta_{j}$, then

$$
\alpha_{1}=\cdots=\alpha_{h}=\{1, \cdots, n\}, \quad \alpha_{h+1}=\cdots=\alpha_{k}=\varnothing ;
$$

if, further, all principal minors of $A$ are positive, we have Marcus' inequality.

The condition $\left|\psi\left(A ; \beta_{1}, \beta_{2}\right)\right| \leqq 1$ is equivalent by (3) to

$$
\left|A\left(\beta_{1} \cap \beta_{2}\right) A\left(\beta_{1} \cup \beta_{2}\right)\right| \leqq\left|A\left(\beta_{1}\right) A\left(\beta_{2}\right)\right|,
$$

which is known to hold for positive definite, totally positive, and $M$-matrices (cf. [3]). If all principal minors of $A$ are positive, a characterization of all matrices satisfying the hypotheses of Theorem 1 is given in [1].

TheOREM 2. Suppose all principal minors of $A$ and $B$ are nonzero, and $\left|\psi\left(A ; \beta_{1}, \beta_{2}\right)\right| \leqq\left|\psi\left(B ; \beta_{1}, \beta_{2}\right)\right|$ for all $\beta_{1}, \beta_{2}$. Then, for all $\beta_{1}, \cdots, \beta_{k}$,

$$
\left|\psi\left(A ; \beta_{1}, \cdots, \beta_{k}\right)\right| \leqq\left|\psi\left(B ; \beta_{1}, \cdots, \beta_{k}\right)\right| \text {. }
$$

To obtain Fan's theorem, we need to use Proposition 3 of [3] (which gives the case $k=2$ under the hypotheses of Theorem $\mathrm{B}$ ) and a remark which follows easily from two theorems of Ostrowski ([7, Satz I and III]; see also [3]):

REMARK. Let $A$ and $B$ satisfy the hypotheses of Theorem B. If $\alpha \subset \beta$ are subsets of $\{1, \cdots, n\}$, then

$$
A(\beta) / A(\alpha) \leqq|B(\beta) / B(\alpha)| \text {. }
$$

Under the hypotheses of Theorem $\mathrm{B}$, we have $\alpha_{i} \subseteq\{1, \cdots, n\}$, $i=1, \cdots, h, \alpha_{i}=\varnothing, i=h+1, \cdots, k$. Therefore 


$$
\phi\left(A ; \beta_{1}, \cdots, \beta_{k}\right)=\psi\left(A ; h ; \beta_{1}, \cdots, \beta_{k}\right) \prod_{i=1}^{h} \frac{\operatorname{det} A}{A\left(\alpha_{i}\right)} ;
$$

the conclusion of Fan's Theorem now follows immediately.

Given $\beta_{1}, \cdots, \beta_{k}$, subsets of $\{1, \cdots, n\}$, we define

$$
\gamma_{i}=\bigcap_{i \in \beta_{j}} \beta_{j}, \quad i=1, \cdots, n .
$$

If we assume with Marcus that each $i, i=1, \cdots, n$, occurs in exactly $h$ of the $\beta_{j}$, then it is easy to show that, for $i \neq j$, either $\gamma_{i}=\gamma_{j}$ or $\gamma_{i} \cap \gamma_{j}=\varnothing$; clearly, $U \gamma_{i}=\{1, \cdots, n\}$. We can now determine the case of equality in Marcus' theorem. $A[\gamma]$ denotes the principal submatrix of $A$ on the rows and columns indexed by $\gamma$.

THEOREM 3. Under the hypotheses of Theorem A, we have equality in (1) if and only if $A$ is a "direct sum" of the $A\left[\gamma_{g}\right]$ (i.e. $a_{i j}=0$ unless $\{i, j\} \subset \gamma_{o}$ for some $g$ ).

3. Proof of Theorem 1. By hypotheses the theorem is true for $k=2$; assume it is true for $2, \cdots, k-1$. Let $Q_{h k}$ be the collection of subsets with $h$ elements from $\{1, \cdots, k\}$. It is easy to see that

$$
\alpha_{h}=\bigcup_{\omega \in Q_{h k}}\left(\bigcap_{i \in \omega} \beta_{i}\right)
$$

and $\alpha_{1} \supseteq \alpha_{2} \supseteq \ldots \supseteq \alpha_{k}$. Now we define

$$
\alpha_{h^{\prime}}=\underset{\omega^{\prime} \in Q_{h, k-1}}{U}\left(\bigcap_{i \in \omega^{\prime}} \beta_{i}\right) \text {; }
$$

$\alpha_{1}^{\prime} \supseteq \alpha_{2}^{\prime} \supseteq \cdots \supseteq \alpha_{k-1}^{\prime}$ and $\alpha_{h} \supseteq \alpha_{h}^{\prime}$ for $h=1, \cdots, k-1$. Note that

$$
\begin{aligned}
\alpha_{k-1}^{\prime} \cap \beta_{k} & =\alpha_{k} \\
\alpha_{h}^{\prime} \cap\left(\alpha_{h+1}^{\prime} \cup \beta_{k}\right) & \left.=\alpha_{h+1}^{\prime} \cup\left(\alpha_{h}^{\prime} \cap \beta_{k}\right)=\alpha_{h+1}\right) \\
\alpha_{h}^{\prime} \cup\left(\alpha_{h+1}^{\prime} \cup \beta_{k}\right) & =\alpha_{h}^{\prime} \cup \beta_{k} \\
\alpha_{1}^{\prime} \cup \beta_{k} & =\alpha_{1} .
\end{aligned}
$$

Now by induction and the result for $h=2$,

$$
\begin{aligned}
&\left|A\left(\beta_{1}\right) \cdots A\left(\beta_{k-1}\right) A\left(\beta_{k}\right)\right| \geqq\left|A\left(\alpha_{1}^{\prime}\right) \cdots A\left(\alpha_{k-1}^{\prime}\right) A\left(\beta_{k}\right)\right| \\
& \geqq\left|A\left(\alpha_{1}^{\prime}\right) \cdots A\left(\alpha_{k-2}^{\prime}\right) A\left(\alpha_{k-1}^{\prime} \cup \beta_{k}\right) A\left(\alpha_{k}\right)\right| \geqq \cdots \\
& \geqq\left|A\left(\alpha_{1}\right) \cdots A\left(\alpha_{k}\right)\right|,
\end{aligned}
$$

which is equivalent to (4). 
4. Proof of Theorem 2. By hypothesis the theorem is true for $k=2$; assume it true for $2, \cdots, k-1$. Now

$$
\begin{aligned}
&\left|\frac{\prod_{h=1}^{k} A\left(\alpha_{h}\right)}{\prod_{h=1}^{k} A\left(\beta_{h}\right)}\right|=\left|\frac{\prod_{h=1}^{k} A\left(\alpha_{h}\right) \prod_{h=1}^{k-1} A\left(\alpha_{h}^{\prime}\right) \prod_{h=2}^{k-2} A\left(\alpha_{h}^{\prime} \cup \beta_{k}\right)}{\prod_{h=1}^{k} A\left(\beta_{h}\right) \prod_{h=1}^{k-1} A\left(\alpha_{h}^{\prime}\right) \prod_{h=2}^{k-2} A\left(\alpha_{h}^{\prime} \cup \beta_{k}\right)}\right| \\
&=\mid \frac{A\left(\alpha_{k}\right) A\left(\alpha_{k-1}^{\prime} \cup \beta_{k}\right)}{A\left(\alpha_{k-1}^{\prime}\right) A\left(\beta_{k}\right)} \prod_{h=2}^{k-2} \frac{A\left(\alpha_{h+1}\right) A\left(\alpha_{h}^{\prime} \cup \beta_{k}\right)}{A\left(\alpha_{h}^{\prime}\right) A\left(\alpha_{h+1}^{\prime} \cup \beta_{k}\right)} \\
& \cdot \frac{A\left(\alpha_{2}\right) A\left(\alpha_{1}\right)}{A\left(\alpha_{1}^{\prime}\right) A\left(\alpha_{2}^{\prime} \cup \beta_{k}\right)} \prod_{h=1}^{k-1} \frac{A\left(\alpha_{h}^{\prime}\right)}{A\left(\beta_{h}\right)} \mid
\end{aligned}
$$

and by the inductive hypothesis, multiplying inequalities for the corresponding factors for $A$ and $B$, we are done.

Proof of Theorem 3. Suppose $\phi\left(A ; h ; \beta_{1}, \cdots, \beta_{k}\right)=1$; under the hypotheses of Theorem $\mathrm{A}$, this is equivalent to $\psi\left(A ; \beta_{1}, \cdots, \beta_{k}\right)=1$. It is sufficient to prove that if $\gamma=\gamma_{1}=\{1, \cdots, p\}=\bigcap_{j=1}^{h} \beta_{j}$, then $a_{i o}=0$ if $i \leqq p, g>p$ or $i>p, g \leqq p$. But now for each $g_{0}>p$, there exists a $\beta_{i}, i>h$; say $i=h+1$, such that $g_{0} \in \beta_{h+1}, \gamma \cap \beta_{h+1}=\varnothing$. If we consider the inductive procedure we used in Theorem 1, at some stage in the chain of inequalities (we may omit the absolute value signs)

$$
A\left(\beta_{1}\right) \cdots A\left(\beta_{k}\right) \geqq \cdots \geqq A\left(\alpha_{1}\right) \cdots A\left(\alpha_{k}\right),
$$

we have, as $\beta_{1} \cap \cdots \cap \beta_{h}=\gamma$ and $\gamma \cap \beta_{h+1}=\varnothing$,

$$
\begin{aligned}
A\left(\beta_{1} \cup \cdots \cup \beta_{h}\right) \cdots A(\gamma) A\left(\beta_{h+1}\right) \cdots A\left(\beta_{k}\right) & \\
& \geqq A\left(\beta_{1} \cup \cdots \cup \beta_{h}\right) \cdots A\left(\gamma \cup \beta_{h+1}\right) \cdots A\left(\beta_{k}\right) .
\end{aligned}
$$

If $\phi\left(A ; h ; \beta_{1}, \cdots, \beta_{k}\right)=1$, we must have

$$
A(\gamma) A\left(\beta_{h+1}\right)=A\left(\gamma \cup \beta_{h+1}\right),
$$

which implies (cf. [5, Vol. I, p. 255]) $a_{i g_{0}}=a_{g_{0} i}=0$ for $i \leqq p(i \in \gamma)$.

Suppose $A$ is the "direct sum" of the $A\left[\gamma_{i}\right]$. Each $\beta_{j}$ and hence each $\alpha_{i}$ is a union of $\gamma_{0}$; thus

$$
A\left(\beta_{j}\right)=\prod_{\gamma_{0} \subset \beta_{i}} A\left(\gamma_{0}\right), \quad A\left(\alpha_{i}\right)=\prod_{\gamma_{0} \subset \alpha_{i}} A\left(\gamma_{0}\right) .
$$

Since each index occurs $h$ times, each $A\left(\gamma_{g}\right)$ occurs $h$ times (both in the numerator and denominator of $\left.\phi\left(A ; h ; \beta_{1}, \cdots, \beta_{k}\right)\right)$. Thus we have equality in (1). 
5. Remarks. Classical determinantal inequalities have recently been generalized in two different ways. In the first, Marvin Marcus and others have considered "generalized matrix functions" (cf. [6], [10], [11]). The author thanks Marvin Marcus and the referee for pointing out (independently) that this note is related to [11]. The inequality (13) of Theorem 5 of [11] is, like Theorem 3 of [6], sharpened by Theorem 1 .

In the second type of generalization, Ky Fan considers real-valued functions $f$ on a distributive lattice $L$ which are "subadditive": $f(\alpha \wedge \beta)+f(\alpha \bigvee \beta) \leqq f(\alpha)+f(\beta)$ for all $\alpha, \beta \in L$. For matrices $A$ with positive principal minors and satisfying the hypotheses of Theorem 1 , the function $f(\alpha)=\log A(\alpha)$ is subadditive on the lattice of subsets of $\{1, \cdots, n\}$. In this setting Fan has generalized a classical identity of Szász [9], [12] and (after seeing a preprint of this note) unified and generalized our Theorems 1 and 2 [8].

\section{REFERENCES}

1. David Carlson, Weakly sign-symmetric matrices and some determinantal inequalities, Colloq. Math. 17 (1967), 123-127.

2. Ky Fan, Inequalities for M-matrices, Proc. Konig Nederl. Akad. Wetensch. Amsterdam Ser. A 67 (1964), 602-610.

3. - Note on M-matrices, Quart. J. Math. Oxford (2) 11 (1960), 43-49.

4. - Some matrix inequalities, Abh. Math. Sem. Univ. Hamburg 29 (1966), $185-196$.

5. F. R. Gantmacher, The theory of matrices, Chelsea, New York, 1959.

6. Marvin Marcus, Matrix applications of a quadratic identity for decomposable symmetrized tensors, Bull. Amer. Math. Soc. 71 (1965), 360-364.

7. Alexander Ostrowski, Über die Determinanten mit überwiegender Hauptdiagonale, Comment. Math. Helv. 10 (1937), 69-96.

8. Ky Fan, An inequality for subadditive functions on a distributive lattice, Linear Algebra Appl. (to appear).

9. - Subadditive functions on a distributive lattice and an extension of Szasz's inequality, J. Math. Anal. Appl. (to appear).

10. Marvin Marcus and Henryk Minc, Generalized matrix functions, Trans. Amer. Math. Soc. 116 (1965), 316-329.

11. Marvin Marcus and George Soules, Some inequalities for combinatorial matrix functions, J. Combinatorial Theory 2 (1967), 145-163.

12. O. Szász, Über eine Verallgemeinerung des Hadamardschen Determinantensatzes, Monatsh. Math. Phys. 28 (1917), 253-257.

Oregon State University 